\title{
生物质炭添加对毛竹林土壤呼吸动态和温度敏感 性的影响
}

葛晓改 ${ }^{1,2}$ 周本智 ${ }^{1,2}$ 肖文发 ${ }^{3}$ 王小明 1,2 曹永慧 1,2 叶 明 4

${ }^{1}$ 中国林业科学研究院亚热带林业研究所, 杭州 $311400 ;{ }^{2}$ 国家林业局钱江源森林生态系统定位观测研究站, 杭州 $311400 ;{ }^{3}$ 中国林业科学研究院森林 生态环境与保护研究所, 国家林业局森林生态环境重点实验室, 北京 $100091 ;{ }^{4}$ 建德市寿昌林场, 浙江寿昌 311600

摘 要 为探讨生物质炭添加对森林原位土壤呼吸动态及温度敏感性的影响, 于2014年5月至2016年4月对浙江省杭州市富 阳区庙山坞林区毛竹(Phyllostachys edulis) 林进行了为期两年的生物质炭添加试验, 生物质炭施加量分别为0 (CK)、 5 (LB)、 $10(\mathrm{MB})$ 和 $20 \mathrm{t} \cdot \mathrm{hm}^{-2}(\mathrm{HB})$ 。利用LI-8100土壤碳通量系统测定土壤呼吸速率时空动态。结果表明: 添加生物质炭会降低毛竹林 土壤呼吸速率且呈现明显的季节动态, 土壤呼吸速率在6-7月最高(林分 1中LB处理除外), 1月或2月最低, 添加生物质炭对毛 竹林土壤呼吸的影响显著; CK、 $\mathrm{LB} 、 \mathrm{MB}$ 和HB处理的年平均土壤呼吸速率分别为 $3.32 、 2.66 、 3.04$ 和 $3.24 \mu \mathrm{mol} \cdot \mathrm{m}^{-2} \cdot \mathrm{s}^{-1}$; 与对 照相比, LB、MB和HB处理下年平均土壤呼吸速率分别降低 $2.33 \%-54.72 \% 、 1.28 \%-44.21 \%$ 和 $0.09 \%-39.22 \%$ 。添加生物质炭 使LB、MB、HB处理的土壤水分含量分别增加了 $0.97 \%-75.58 \% 、 0.87 \%-48.18 \%$ 和 $0.68 \%-74.73 \%$ 。土壤呼吸速率与 $5 \mathrm{~cm}$ 土壤 温度呈现显著的指数相关关系, 与 $5 \mathrm{~cm}$ 土壤水分含量没有显著相关性, 但与温度和水分呈显著相关关系。生物质炭添加影响 土壤呼吸温度敏感性, LB、MB处理明显增加土壤温度敏感性。LB、MB和 $\mathrm{HB}$ 处理下年平均累积土壤呼吸 $\mathrm{CO}_{2}$ 排放分别降低 $7.98 \%-35.09 \%$ 、 $1.48 \%-20.63 \%$ 和 $-4.71 \%-7.68 \%$ 。添加生物质炭显著降低毛竹林土壤碳排放和土壤温度敏感性, 对缓解气候 变化具有一定意义。

关键词＼cjkstart生物质炭添加; 土壤呼吸; 毛竹; 温度敏感性; 土壤温度; 土壤含水量

引用格式: 葛晓改, 周本智, 肖文发, 王小明, 曹永慧, 叶明 (2017). 生物质炭添加对毛竹林土壤呼吸动态和温度敏感性的影响. 植物生态学报, 41, 1177-1189. doi: 10.17521/cjpe.2017.0098

\section{Effects of biochar addition on dynamics of soil respiration and temperature sensitivity in a Phyllostachys edulis forest}

GE Xiao-Gai ${ }^{1,2}$, ZHOU Ben-Zhi ${ }^{1,2^{*}}$, XIAO Wen-Fa ${ }^{3}$, WANG Xiao-Ming ${ }^{1,2}$, CAO Yong-Hui ${ }^{1,2}$, and YE Ming ${ }^{4}$

${ }^{1}$ Research Institute of Subtropical Forestry, Chinese Academy of Forestry, Hangzhou 311400, China; ${ }^{2}$ Qianjiangyuan Forest Ecosystem Research Station, State Forestry Administration, Hangzhou 311400, China; ${ }^{3}$ State Forestry Administration Key Laboratory of Forest Ecology and Environment; Research Institute of Forest Ecology, Environment and Protection, Chinese Academy of Forestry, Beijing 100091, China; and ${ }^{4}$ Shouchang Forest Center of Jiande, Shouchang, Zhejiang 311600, China

\section{Abstract}

Aims Recent studies have shown that artificial addition of biochar is an effective way to mitigate atmospheric carbon dioxide concentrations. However, it is still unclear how biochar addition influences soil respiration in Phyllostachys edulis forests of subtropical China. Our objectives were to examine the effects of biochar addition on the dynamics of soil respiration, soil temperature, soil moisture, and the cumulative soil carbon emission, and to determine the relationships of soil respiration with soil temperature and moisture.

Methods We conducted a two-year biochar addition experiment in a subtropical P. edulis forest from 2014.05 to 2016.04. The study site is located in the Miaoshanwu Nature Reserve in Fuyang district of Hangzhou, Zhejiang Province, in southern China. The biochar addition treatments included: control (CK, no biochar addition), low rate of biochar addition (LB, $\left.5 \mathrm{t} \cdot \mathrm{hm}^{-2}\right)$, medium rate of biochar addition $\left(\mathrm{MB}, 10 \mathrm{t} \cdot \mathrm{hm}^{-2}\right)$, and high rate of biochar addition ( $\mathrm{HB}, 20 \mathrm{t} \cdot \mathrm{hm}^{-2}$ ). Soil respiration was measured by using a LI-8100 soil $\mathrm{CO}_{2}$ efflux system.

Important findings Soil respiration was significantly reduced by biochar addition, and exhibited an apparent seasonal pattern, with the maximum occurring in June or July (except LB in one of the replicated stand) and the minimum in January or February. There were significant differences in soil respiration between the CK and the

收稿日期Received: 2017-04-10 接受日期Accepted: 2017-08-29

* 通信作者Author for correspondence (E-mail: benzhi_zhou@126.com) 
treatments. Annual mean soil respiration rate in the $\mathrm{CK}, \mathrm{LB}, \mathrm{MB}$ and $\mathrm{HB}$ were 3.32, 2.66, 3.04 and $3.24 \mu \mathrm{mol} \cdot \mathrm{m}^{-2} \cdot \mathrm{s}^{-1}$, respectively. Compared with CK, soil respiration rate was $2.33 \%-54.72 \%$ lower in the LB, $1.28 \%-44.21 \%$ lower in the MB, and $0.09 \%-39.22 \%$ lower in the HB. The soil moisture content was increased by $0.97 \%-75.58 \%$ in LB, $0.87 \%-48.18 \%$ in MB, and $0.68 \%-74.73 \%$ in HB, respectively, compared with CK. Soil respiration exhibited a significant exponential relationship with soil temperature and a significant linear relationship with combination of soil temperature and moisture at the depth of $5 \mathrm{~cm}$; no significant relationship was found between soil respiration and soil moisture alone. The temperature sensitivity $\left(Q_{10}\right)$ value was reduced in LB and HB. Annual accumulative soil carbon emission in the LB, MB and HB was reduced by $7.98 \%-35.09 \%$, $1.48 \%-20.63 \%$, and $-4.71 \%-7.68 \%$, respectively. Biochar addition significantly reduced soil carbon emission and soil temperature sensitivity, highlighting its role in mitigating climate change.

Key words biochar addition; soil respiration; Phyllostachys edulis; temperature sensitivity; soil temperature; soil moisture

Citation: Ge XG, Zhou BZ, Xiao WF, Wang XM, Cao YH, Ye M (2017). Effects of biochar addition on dynamics of soil respiration and temperature sensitivity in a Phyllostachys edulis forest. Chinese Journal of Plant Ecology, 41, 1177-1189. doi: $10.17521 /$ cjpe. 2017.0098

大气中温室气体 $\mathrm{CO}_{2}$ 浓度升高导致的全球变暖 及一系列其他生态问题已引起世界各国的高度关注, 成为科学研究领域的热点问题(Marland \& Marland, 2003; 徐涌, 2012; Mitchell et al., 2015)。森林作为陆 地生态系统的主体, 在减缓大气 $\mathrm{CO}_{2}$ 浓度升高调节 全球碳平衡方面有着重要作用; 在森林生态系统中, 生物质炭是森林火干扰的主要产物, 影响土壤物 理、化学和生物过程, 在多数森林生态系统中很普 遍, 但生物质炭的生物功能在森林生态系统中却被 忽略(DeLuca et al., 2006)。有研究证实, 生物质炭具 有高度稳定性和较强的吸附性能, 因而可长期存在 于环境中, 在稳定土壤有机碳库、增加土壤碳库容 量、减缓土壤碳释放方面有重要意义(Lal et al., 2007), 在应对全球气候变化及环境系统中发挥着 重要作用(Sohi et al., 2010)。目前, 生物质炭添加因 能增加植物生产力和作物产量, 在农业系统中广泛 应用, 而在林业上的应用关注较少(Mitchell et al., 2015)。然而, 森林土壤中添加生物质炭亦能增加森 林生产力(Thomas \& Gale, 2015), 更重要的是, 生 物质炭有潜在的碳吸存能力并能减缓土壤 $\mathrm{CO}_{2}$ 排 放。因此, 生物质炭在林业上的应用研究很有必要, 添加生物质炭对森林土壤碳矿化、吸存及土壤物理 化学性质的影响将日益受到关注。

研究表明生物质炭添加明显影响原位土壤有机 碳矿化(Herath et al., 2015)。土壤呼吸速率是研究土 壤碳矿化的重要指标, 是陆地生态系统碳循环的重 要过程, 全球年平均土壤呼吸通量为 $91 \mathrm{Pg} \cdot \mathrm{a}^{-1}$ (1965-2012 年为 87-95 $\left.\mathrm{Pg} \cdot \mathrm{a}^{-1}\right)$ (Hashimoto et al., 2015), 因此, 土壤呼吸的强弱在很大程度上决定了
全球气候变化与碳循环间的反馈关系(葛晓改等, 2016)。生物质炭对原位土壤有机碳排放的影响存在 不确定性(He et al., 2016): 一方面, 生物质炭添加 促进原位土壤碳矿化(正激发效应), 这可能诱发土 壤理化性质的改变或生物质炭自身碳矿化(Wardle et al., 2008)或为微生物提供养分而增加微生物活性; 另一方面, 生物质炭添加抑制原位土壤碳矿化(负 激发效应), 生物质炭表面吸附原位土壤易溶物或 促进土壤团聚体及原位土壤有机碳的稳定性 (Herath et al., 2015)。研究表明生物质炭的正激发效 应随着时间会降低, 甚至转化成负激发效应 (Zimmerman et al., 2011)。目前, 生物质炭添加实验 大多基于室内培养或短期的野外试验研究, 培养试 验过程通常是在最优化状态下进行, 如常温状态和 田间持水量在 $60 \%-70 \%$ 的情况不能代表野外干湿 状态下真实的土壤呼吸(Yuste et al., 2007); 生物质 炭添加增加土壤水分含量且明显影响土壤呼吸的温 度敏感性 $\left(Q_{10}\right)(\mathrm{He}$ et al., 2016)。因此, 有必要开展野 外生物质炭添加对土壤呼吸激发效应的大小、持久 性和温度敏感性的相关研究。

竹林是我国重要的森林资源类型，中国现有竹 林面积约 601 万 $\mathrm{hm}^{2}$, 且具有生长速度快、隔年连续 采伐和可长期利用等特点, 每年有大量的新笋成竹 向周围扩展, 因而竹林固定 $\mathrm{CO}_{2}$ 的潜力巨大, 对吸 存大气中的 $\mathrm{CO}_{2}$ 具有重要作用。然而, 中国每年竹材 砍伐量为 $800-900$ 万 $\mathrm{t}$, 相当于 1000 余万 $\mathrm{m}^{3}$ 木材, 但 在竹材被砍伐加工利用的同时，38\%-84\%的生物质 废弃物在燃烧过程中被释放(Hughes et al., 1999)。如 果地上竹材加工和砍伐的副产品炭化成生物质炭并 
应用到土壤，这些废弃物中 $50 \%$ 以上的碳将以高度 稳定的形式被吸存在土壤中(Lehmann et al., 2006), 对全球碳循环和缓解全球气候变化具有重要意义 (Forbes et al., 2006)。本研究以浙江省广泛分布的毛 竹(Phyllostachys edulis) 林为研究对象, 通过生物质 炭添加试验研究不同毛竹林分 2 年的土壤呼吸月动 态及其温度、水分含量响应, 以探讨和验证生物质 炭添加对森林土壤呼吸激发效应的方向、大小、持 久性及其温度敏感性, 为竹林土壤碳排放的激发效 应研究的系统性、完整性和深入性奠定科学基础, 对生物质炭输入的变化规律、稳定机理及其与土壤 相互作用的深入研究有重要意义。

\section{1 材料和方法}

\section{1 试验地区概况}

研究区域位于浙江省杭州市富阳区国家林业局 钱江源森林生态系统定位研究站庙山坞林区, 地理 位置为 $119.93^{\circ}-120.03^{\circ} \mathrm{E}, 30.05^{\circ}-30.10^{\circ} \mathrm{N}$ 。庙山坞 自然保护林区面积约 $536.7 \mathrm{hm}^{2}$, 核心区面积 $348.8 \mathrm{hm}^{2}$, 海拔11-536 m。保护区内土壤类型有红 壤、黄红壤、幼红壤, 红壤面积约占保护区面积的 $61 \%$, 土壤 $\mathrm{pH}$ 值平均为 5.72 。研究区具有典型的亚 热带季风气候特征, 年平均气温为 $16.9{ }^{\circ} \mathrm{C}$, 最热月 平均气温 $25.4{ }^{\circ} \mathrm{C}$, 最冷月平均气温 $4.5{ }^{\circ} \mathrm{C}$ (周本智, 2006)。夏春秋冬, 雨量依次减少, 平均年降水量为 $1513 \mathrm{~mm}$ 。年日照时间达 $1995 \mathrm{~h}$, 全年无霜期达 237天。

研究区地带性植被为常绿阔叶林, 由于过去用 材和薪炭的需求, 以及农业活动的发展, 地带性植 被已被破坏, 现在主要植被类型包括毛竹林、杉木 (Cunninghamia lanceolata)林、马尾松(Pinus massoniana) 林、天然次生林、灌木林等, 其中人工毛竹 林占总面积的 $30 \%$ 。

\section{2 样地布置与土壤呼吸测定}

2014年4月, 随机选择立地条件相似的毛竹林 4
块, 每块林分随机各设置 3 个 $20 \mathrm{~m} \times 30 \mathrm{~m}$ 的样地, 每个样地中随机设置 4 个 $1 \mathrm{~m} \times 1 \mathrm{~m}$ 的小样方, 用于 生物质炭添加处理, 每处理3次重复。为防止相互干 扰，小样方间至少相隔 $10 \mathrm{~m}$ 。根据国内外生物质炭 添加试验研究的强度和竹林经营实况(Tammeorg et $a l .$, 2014), 生物质炭添加试验分 4 个处理, 分别为对 照 $(\mathrm{CK})$ 、低生物质炭( $\left.\mathrm{LB}, 5 \mathrm{t} \cdot \mathrm{hm}^{-2}\right)$ 、中生物质炭( $\mathrm{MB}$, $10 \mathrm{t} \cdot \mathrm{hm}^{-2}$ )和高生物质炭处理( $\left.\mathrm{HB}, 20 \mathrm{t} \cdot \mathrm{hm}^{-2}\right)$ 。样地基 本概况见表1。

背景值调查: 对样方内的毛竹进行每木检尺和 样地植被调查, 每样方分别用直径为 $5 \mathrm{~cm}$ 的土钻沿 $\mathrm{S}$ 形采深度为 $0-10 \mathrm{~cm}$ 表层土各取 9 钻, 任意 3 钻的同 层土壤分别合并, 每土样重复3次。同时每个小样方 各层同步取环刀样品, 每层共 9 次重复。进行土壤总 有机碳、总氮和总磷测定。

生物质炭添加采用平铺法。在上述试验区样地 外选择环境条件和土壤状况与样地相似的毛竹林, 挖取 0-15 $\mathrm{cm}$ 深的土壤约 $100 \mathrm{~kg}$ (土壤容重为 $1.04 \mathrm{~g} \cdot \mathrm{kg}^{-1}$ ), 剔除石砾等杂质, 风干过篮后混匀约 $60 \mathrm{~kg}$, 分成4份, 每份约 $15 \mathrm{~kg}$ 。4份土壤与生物质炭 分别按 $0 、 0.32 \% 、 0.64 \%$ 和 $1.28 \%$ 混合备用(分别对 应上述对照、低生物质炭、中生物质炭和高生物质 炭处理), 以防生物质炭流失。在选定的样方中分别 添加上述对应的混合土(约 $\left.1 \mathrm{~kg} \cdot \mathrm{m}^{-2}\right)$ 。同时测定风干 土有机碳含量, 每林分 3 次重复。供试生物质炭是在 $450{ }^{\circ} \mathrm{C}$ 条件下炭化所得, 炭粒直径在1-2 mm间(分 别过 $1 \mathrm{~mm}$ 和 $2 \mathrm{~mm}$ 篮)。

2014年4月, 分别在设定的毛竹林样地各处理 小样方内随机布设 3 个聚氯乙烯( $\mathrm{PVC}$ ) 土壤环(内径 $20 \mathrm{~cm}$, 高 $10 \mathrm{~cm}$, 埋入土壤7-8 cm)。土壤呼吸用 LI-8100便携式土壤碳通量测定系统(LI-COR, Nebraska, USA)进行测定, 用其自带的土壤温度、水分 探头测 $5 \mathrm{~cm}$ 深度的土壤温度和湿度 $($ 土壤体积含水 量)。从2014年5月至2016年4月, 于每月中旬选择晴 朗天气测定。

表1 研究林分特征(平均值土标准偏差)

Table 1 General characteristics of the forest stands studied ( (mean $\pm S D)$

\begin{tabular}{|c|c|c|c|c|c|c|c|c|c|}
\hline $\begin{array}{l}\text { 林分 } \\
\text { Stand }\end{array}$ & $\begin{array}{c}\text { 海拔 } \\
\text { Elevation (m) }\end{array}$ & $\begin{array}{c}\text { 树高 } \\
\text { Tree height (m) }\end{array}$ & $\begin{array}{c}\text { 胸径 } \\
D B H(\mathrm{~cm})\end{array}$ & $\begin{array}{c}\text { 坡度 } \\
\text { Slope }\left(^{\circ}\right)\end{array}$ & $\begin{array}{c}\text { 坡向 } \\
\text { Aspect }\end{array}$ & $\begin{array}{c}\text { 凋落物层厚度 } \\
\operatorname{LLD}(\mathrm{cm})\end{array}$ & $\begin{array}{c}\text { 土壤有机碳 } \\
\operatorname{SOC}\left(\mathrm{g} \cdot \mathrm{kg}^{-1}\right)\end{array}$ & $\begin{array}{c}\text { 总氮 } \\
\mathrm{TN}\left(\mathrm{g} \cdot \mathrm{kg}^{-1}\right)\end{array}$ & $\begin{array}{c}\text { 总磷 } \\
\mathrm{TP}\left(\mathrm{g} \cdot \mathrm{kg}^{-1}\right)\end{array}$ \\
\hline 1 & 141 & 12.8 & 10.2 & 10 & $\mathrm{~S}$ & 2.04 & $33.10 \pm 9.06$ & $2.86 \pm 0.79$ & $0.56 \pm 0.93$ \\
\hline 2 & 115 & 13.0 & 10.3 & 15 & S & 2.22 & $30.1 \pm 8.04$ & $2.59 \pm 0.48$ & $0.37 \pm 1.03$ \\
\hline 3 & 86 & 13.3 & 10.7 & 20 & $\mathrm{~S}$ & 2.47 & $28.8 \pm 8.21$ & $2.38 \pm 0.29$ & $0.43 \pm 1.09$ \\
\hline 4 & 157 & 12.2 & 10.1 & 20 & $\mathrm{~S}$ & 2.60 & $34.1 \pm 8.04$ & $2.59 \pm 0.29$ & $0.72 \pm 1.09$ \\
\hline
\end{tabular}

$\mathrm{DBH}$, diameter at breast height; LLD, litter layer depth; SOC, soil organic carbon; TN, total nitrogen; TP, total phosphorus. 


\section{3 数据处理}

土壤呼吸速率 $\left(R_{\mathrm{s}}, \mu \mathrm{mol} \cdot \mathrm{m}^{-2} \cdot \mathrm{s}^{-1}\right)$ 与土壤温度的 关系拟合采用如下模型:

$$
R_{s}=a_{1} \mathrm{e}^{a_{2} T}
$$

式中: $T$ 为 $5 \mathrm{~cm}$ 深度土壤温度 $\left({ }^{\circ} \mathrm{C}\right), a_{1}$ 是温度为 $0{ }^{\circ} \mathrm{C}$ 的土壤呼吸速率, $a_{2}$ 为土壤呼吸的温度反应系数。

土壤呼吸的温度敏感性 $\left(Q_{10}\right)$ 采用如下模型:

$$
Q_{10}=\mathrm{e}^{10 a_{2}}
$$

式中: $a_{2}$ 为温度反应系数。

土壤呼吸与土壤湿度的关系采用如下模型 (Saiz et al., 2006):

$$
R_{s}=b_{1}+b_{2} \times W+b_{3} \times W^{2}
$$

式中: $W$ 为土壤湿度 (土壤体积含水量 $\%$ ),$b_{1} 、 b_{2} 、 b_{3}$ 为方程拟合参数。

土壤呼吸与土壤温度和湿度的关系, 采用如下 模型(Saiz et al., 2006):

$$
R_{s}=\left(c_{1} \mathrm{e}^{c_{2} T}\right)\left(c_{3} W+c_{4} W^{2}\right)
$$

式中: $T$ 为 $5 \mathrm{~cm}$ 深度土壤温度 $\left({ }^{\circ} \mathrm{C}\right), W$ 是 $5 \mathrm{~cm}$ 深度土 壤湿度 $(\%), c_{1} 、 c_{2} 、 c_{3} 、 c_{4}$ 为方程拟合参数。

土壤呼吸累积量 $\left(R_{\mathrm{c}}, \mathrm{kg} \cdot \mathrm{m}^{-2} \cdot \mathrm{a}^{-1}\right)$ 计算根据如 下等式:

$$
R_{c}=\sum_{i-1}^{12} R_{\mathrm{si}} \times t_{i} \times 10^{-9} \times 44
$$

式中: $R_{\mathrm{si}}$ 为各个月呼吸速率平均值 $\left(\mu \mathrm{mol} \cdot \mathrm{m}^{-2} \cdot \mathrm{s}^{-1}\right), t_{\mathrm{i}}$ 为各个月时间 $(\mathrm{s}), 44$ 为 $\mathrm{CO}_{2}$ 摩尔质量 $\left(\mathrm{g} \cdot \mathrm{mol}^{-1}\right)$ 。

利用Excel 2007和SPSS 16.0软件进行统计分析 和作图, 生物质炭添加处理下土壤呼吸速率和土壤 呼吸年累积量之间的差异采用单因子方差分析和最 小显著差异法 $(L S D)$ 分析, 显著性水平设定为 $\alpha=$ 0.05 ; 土壤呼吸速率与土壤温度、土壤湿度关系采 用回归分析。

\section{2 结果}

\section{1 生物质炭添加对土壤呼吸季节动态的影响}

生物质炭添加和对照中土壤呼吸均呈明显的季 节变化和单峰模式, 土壤呼吸速率均在6-7月最高 (只有林分 1 的LB处理是 8 月份最高), 1 月或 2 月最低 (图1)。生物质炭添加显著降低了年平均土壤呼吸速 率(林分 3 的HB处理除外) $(p<0.01)$, 毛竹林CK、LB、 $\mathrm{MB}$ 和 $\mathrm{HB}$ 处理的年平均土壤呼吸速率分别为(3.32 $0.16) 、(2.66 \pm 0.45) 、(3.04 \pm 0.18)$ 和 $(3.24 \pm$ $0.19) \mu \mathrm{mol} \cdot \mathrm{m}^{-2} \cdot \mathrm{s}^{-1}$, 即随着添加量的增加土壤呼吸
降低量变小。林分 1 到林分 3 中年平均土壤呼吸速率 均是 $\mathrm{CK}$ 最高, $\mathrm{HB} 、 \mathrm{MB}$ 次之, $\mathrm{LB}$ 最低; 林分 3 的年平 均土壤呼吸速率则是 $H B$ 最高, $C K 、 M B$ 次之, $L B$ 最低。 与对照相比, LB、MB、HB处理下土壤呼吸速率分别 降低 $2.33 \%-54.72 \% 、 1.28 \%-44.21 \%$ 和 $0.09 \%-39.22 \%$ 。

生物质炭添加对生长季(4-10月)和非生长季(11 月到次年3月)土壤呼吸影响显著 $(p<0.01)$ (图 2 ; 表 $2)$, 生物质炭添加处理与季节的交互影响仅林分 1 显著 $(p<0.01)$ (表2)。林分 1 到林分 3 中LB处理均明显 降低生长季土壤呼吸 $(p<0.01)$ 。与对照相比, 林分 1 中生长季的LB、 $\mathrm{MB} 、 \mathrm{HB}$ 分别降低37.02\%、3.06\% 和 $9.69 \%$ ，林分2中分别降低 $25.70 \% 、 19.11 \%$ 和 $5.52 \%$, 林分3中LB处理降低 $7.25 \%, \mathrm{MB}$ 和HB处理分别增加 $0.79 \%$ 和 $5.73 \%$; 林分 4 中 LB、 MB分别降低 $6.19 \%$ 和 $4.09 \%$, HB处理增加 $3.59 \%$ 。非生长季中所有林分的 LB处理、林分 2 和 3 中 MB处理、林分 4 中 $\mathrm{HB}$ 处理较 对照显著降低 $(p<0.01)$ (图2), 1-4林分中LB较对照 分别降低 $27.69 \% 、 32.42 \% 、 17.95 \%$ 和 $18.68 \%$, 林分 2 和 3 中 MB 处理较对照分别降低 $28.77 \%$ 和 $17.78 \%$, 林分4中HB处理比对照降低 $16.22 \%$ 。

\section{2 生物质炭添加对毛竹林土壤温度、水分的影响}

生物质炭添加对土壤水分影响显著, 尤其是 $\mathrm{HB}$ 处理(图3)。在试验的 24 个月中, LB处理中, 林分 1 中仅有 3 个月水分含量增加 $(0.97 \%-17.39 \%)$, 林分 2 中 14 个月水分含量增加 $(1.65 \%-75.58 \%)$, 林分 3 中 12 个月水分含量增加 $(0.87 \%-18.58 \%)$, 林分 4 中有 15 个月水分含量增加 $(0.62 \%-41.49 \%) ; \mathrm{MB}$ 处理中, 林分 1 到林分 4 中分别有 5 个月 $(3.65 \%-16.43 \%) 、 18$ 个月 $(1.59 \%-48.18 \%) 、 13$ 个月 $(0.87 \%-18.58 \%)$ 、9个 月 $(1.11 \%-36.34 \%)$ 水分含量增加; HB处理中, 林分 1 到林分 4 中分别有 13 个月 $(0.89 \%-21.18 \%) 、 21$ 个月 $(2.33 \%-50.40 \%) 、 17$ 个月 $(0.86 \%-74.73 \%)$ 和 17 个月 (0.68\%-39.24\%)水分含量增加。生物质炭添加对毛 竹林土壤温度影响不明显(图4); 最高土壤温度均是 在2015年7月，最低土壤温度是在2015年2月。

\section{3 生物质炭添加下土壤呼吸与土壤温度和水分 的关系}

生物质炭添加下各林分土壤呼吸均与土壤温度 呈显著正相关关系(图5)。基于土壤温度的非线性模 型拟合发现，与对照相比，林分 1 到林分 2 中生物质 炭添加处理后土壤温度对土壤呼吸的解释量 $\left(R^{2}\right)$ 均 降低, 林分 3 和林分 4 (除MB处理)中温度对土壤呼 www.plant-ecology.com 

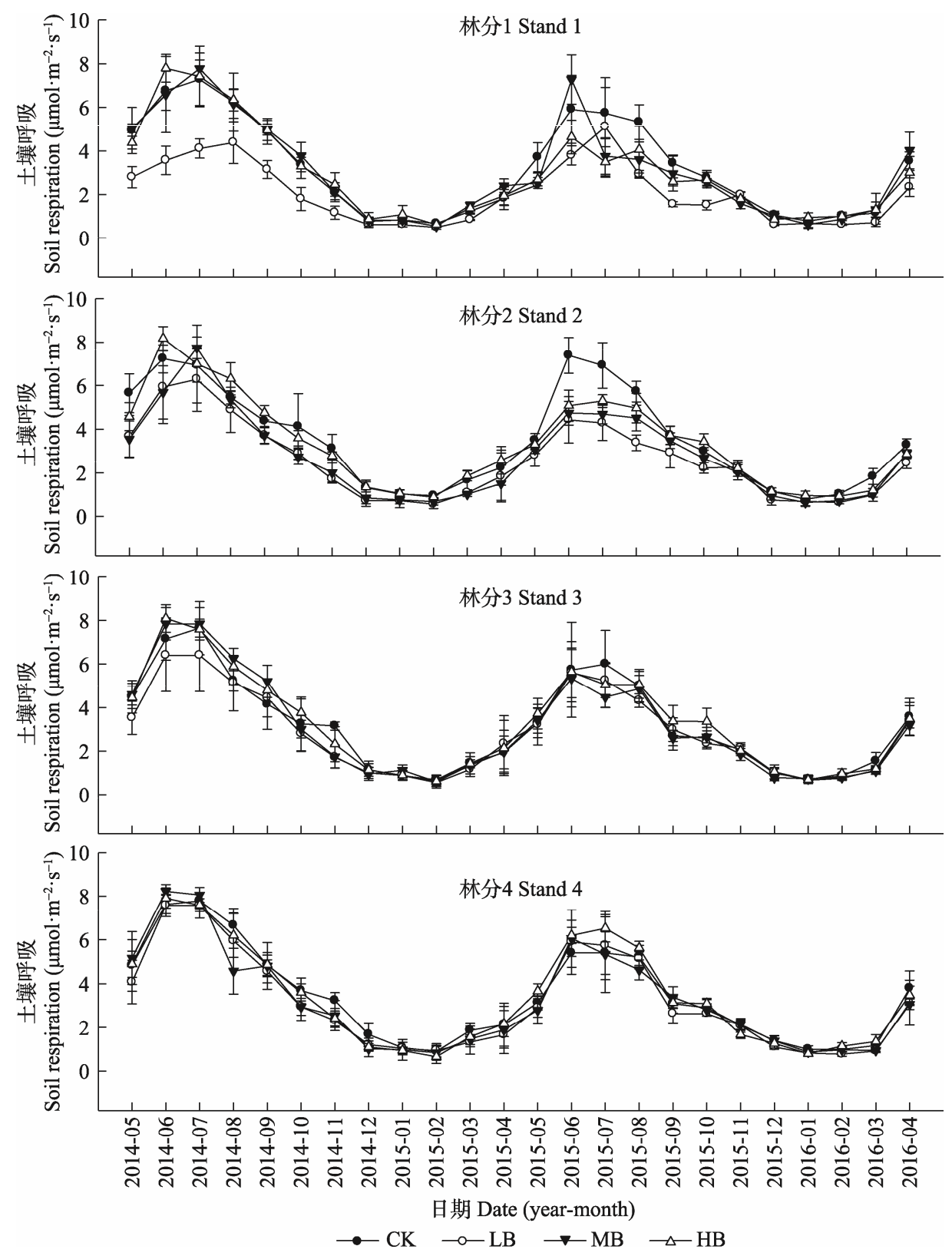

图1 生物质炭添加下毛竹林土壤呼吸月动态(平均值 土标准偏差)。CK, 对照; $\mathrm{LB}$, 低生物质炭施加 $\left(5 \mathrm{t} \cdot \mathrm{hm}^{-2}\right) ; \mathrm{HB}$, 高生物质炭 施加 $\left(20 \mathrm{t} \cdot \mathrm{hm}^{-2}\right) ; \mathrm{MB}$ ，中生物质炭施加 $\left(10 \mathrm{t} \cdot \mathrm{hm}^{-2}\right)$ 。

Fig. 1 Monthly dynamics of soil respiration in Phyllostachys edulis forest stands with different biochar addition treatments (mean \pm $S D)$. CK, control; LB, low rate of biochar addition $\left(5 \mathrm{t} \cdot \mathrm{hm}^{-2}\right)$; HB, high rate of biochar addition $\left(20 \mathrm{t} \cdot \mathrm{hm}^{-2}\right)$; MB, medium rate of biochar addition $\left(10 \mathrm{t} \cdot \mathrm{hm}^{-2}\right)$.

吸变量的解释量 $\left(R^{2}\right)$ 则增加。生物质炭添加处理改 变土壤呼吸的温度敏感性, 与对照相比, 林分 1 中生 物质炭添加处理 $Q_{10}$ 均降低, 林分2仅HB处理 $Q_{10}$ 降 低，林分3中仅LB处理 $Q_{10}$ 降低，林分4中生物质炭 添加处理 $Q_{10}$ 均增加。

生物质炭添加处理后，利用线性模型拟合发现 土壤呼吸与土壤水分含量没有显著相关关系, 但土 壤呼吸与温度、水分交互呈显著的非线性相关关系 $(p<0.01$, 表 3$)$ 。与对照相比, 林分 1 和林分 2 (除LB
处理)中生物质炭添加处理的 $R^{2}$ 值均降低, 林分 3 (除 $\mathrm{LB}$ 处理)和林分 4 (除 $\mathrm{MB}$ 处理)的 $R^{2}$ 值均增加, $\mathrm{CK}$ 处理的土壤温度、水分共同解释土壤呼吸变量的 $72.3 \%-80.2 \%$, LB 处理解释变量的 $68.6 \%-79.5 \%$, MB处理解释变量的 $64.9 \%-75.6 \%$, HB处理解释变 量的 $64.9 \%-85.8 \%$ 。

年平均土壤呼吸的温度敏感性与土壤温度、土 壤含水量的相关关系不显著 $(p>0.05$, 图6), 表明土 壤水分含量增加对温度敏感性 $Q_{10}$ 的变化影响较小。 

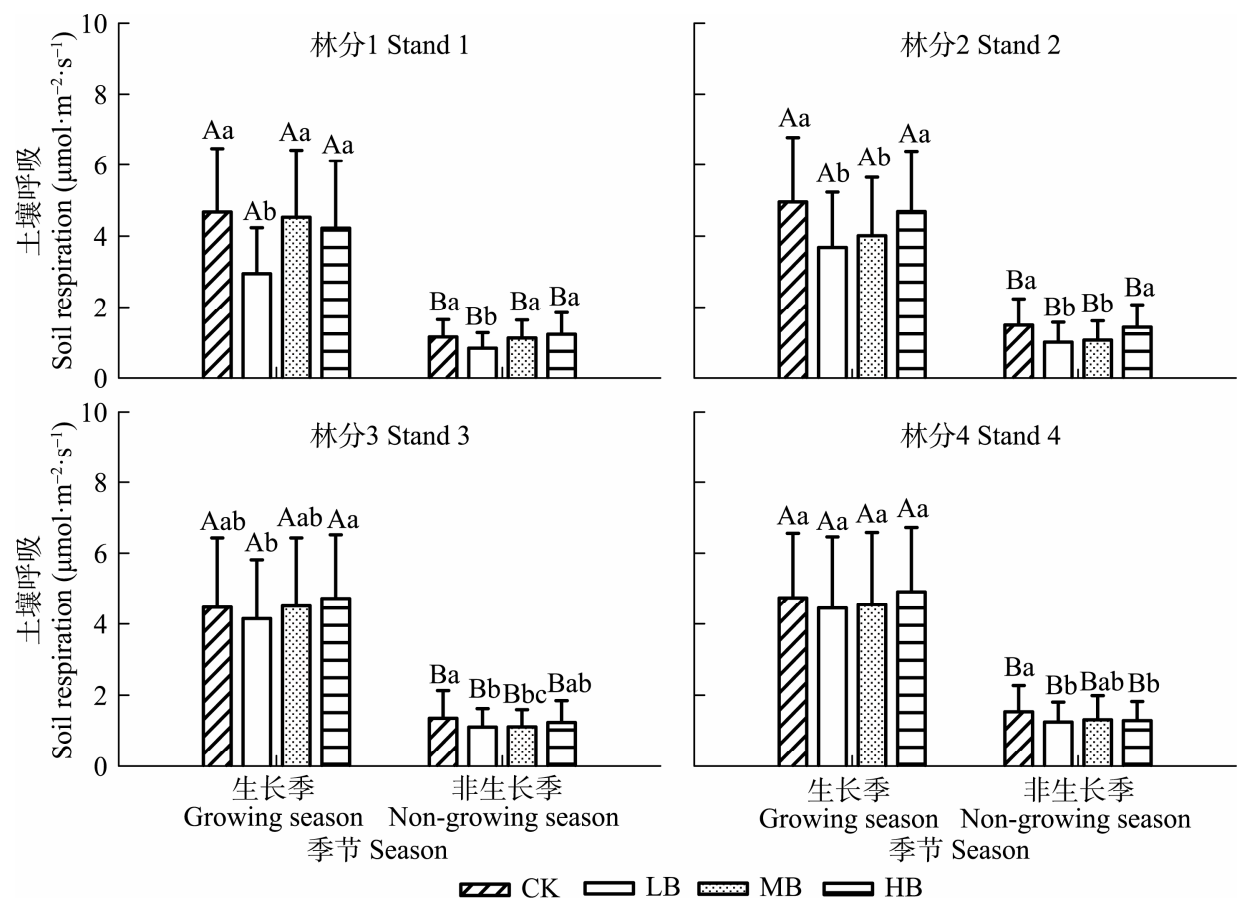

图2 生物质炭添加下毛竹林生长季和非生长季土壤呼吸特征(平均值沶准偏差)。不同小写字母表示相同季节不同生物质炭 处理间差异显著; 不同大写字母表示同一生物质炭处理不同季节差异显著。CK, 对照; LB，低生物质炭施加 $\left(5 \mathrm{t} \cdot \mathrm{hm}^{-2}\right) ; \mathrm{HB}$, 高 生物质炭施加 $\left(20 \mathrm{t} \cdot \mathrm{hm}^{-2}\right)$; MB，中生物质炭施加 $\left(10 \mathrm{t} \cdot \mathrm{hm}^{-2}\right)$ 。

Fig. 2 The characteristics of soil respiration in growing and non-growing seasons in Phyllostachys edulis forest stands with different biochar treatments (mean $\pm S D$ ). Different lowercase letters indicate significant differences among biochar treatments within seasons, and different capital letters indicate significant differences among seasons within biochar treatments. CK, control; LB, low rate of biochar addition $\left(5 \mathrm{t} \cdot \mathrm{hm}^{-2}\right)$; HB, high rate of biochar addition $\left(20 \mathrm{t} \cdot \mathrm{hm}^{-2}\right)$; MB, medium rate of biochar addition $\left(10 \mathrm{t} \cdot \mathrm{hm}^{-2}\right)$.

表2 生物质炭添加和季节对毛竹林土壤呼吸影响的双因子方差分析 Table 2 Two-way ANOVA for the effects of biochar addition treatments and seasons on soil respiration in Phyllostachys edulis forest stands

\begin{tabular}{|c|c|c|c|c|c|c|}
\hline \multirow[t]{2}{*}{$\begin{array}{l}\text { 林分 } \\
\text { Stands }\end{array}$} & \multicolumn{2}{|c|}{$\begin{array}{c}\text { 季节 } \\
\text { Season }\end{array}$} & \multicolumn{2}{|c|}{$\begin{array}{c}\text { 生物质炭添加 } \\
\text { Biochar addition }\end{array}$} & \multicolumn{2}{|c|}{$\begin{array}{c}\text { 季节×生物质炭添加 } \\
\text { Season } \times \text { biochar } \\
\text { addition } \\
\end{array}$} \\
\hline & $F$ & $p$ & $F$ & $p$ & $F$ & $p$ \\
\hline 林分 1 Stand 1 & 673.41 & 0.00 & 16.66 & 0.00 & 7.64 & 0.00 \\
\hline 林分 2 Stand 2 & 731.47 & 0.00 & 13.36 & 0.00 & 2.30 & 0.08 \\
\hline 林分 3 Stand 3 & 695.20 & 0.00 & 1.54 & 0.204 & 0.77 & 0.51 \\
\hline 林分 4 Stand 4 & 655.78 & 0.00 & 1.14 & 0.334 & 0.64 & 0.60 \\
\hline
\end{tabular}

\section{4 生物质炭添加对土壤呼吸年累积碳排放的影响}

生物质炭添加处理降低土壤呼吸年总量(林分3 中HB处理除外)(图7)。各林分土壤呼吸年总量均是 LB处理最低, CK处理最高(林分3中HB处理除外)。 林分 1 中CK中土壤呼吸年总量是 $4.47 \mathrm{~kg} \cdot \mathrm{m}^{-2} \cdot \mathrm{a}^{-1}, \mathrm{LB}$ 是 $2.88 \mathrm{~kg} \cdot \mathrm{m}^{-2} \cdot \mathrm{a}^{-1}, \mathrm{MB}$ 是 $4.33 \mathrm{~kg} \cdot \mathrm{m}^{-2} \cdot \mathrm{a}^{-1}, \mathrm{HB}$ 是 $4.14 \mathrm{~kg} \cdot \mathrm{m}^{-2} \cdot \mathrm{a}^{-1}$, 与对照相比, LB、MB和HB分别降 低 $35.09 \% 、 3.07 \%$ 和 $7.68 \%$; 林分 2 中，LB $(3.58$ $\left.\mathrm{kg} \cdot \mathrm{m}^{-2} \cdot \mathrm{a}^{-1}\right)<\mathrm{MB}\left(3.87 \mathrm{~kg} \cdot \mathrm{m}^{-2} \cdot \mathrm{a}^{-1}\right)<$ HB $(4.63$ $\left.\mathrm{kg} \cdot \mathrm{m}^{-2} \cdot \mathrm{a}^{-1}\right)<\mathrm{CK}\left(4.89 \mathrm{~kg} \cdot \mathrm{m}^{-2} \cdot \mathrm{a}^{-1}\right), \mathrm{LB} 、$ MB和HB处 理的土壤呼吸年总量分别降低 $27.00 \% 、 20.63 \%$ 和 $5.53 \%$; 林分 3 中LB $\left(4.01 \mathrm{~kg} \cdot \mathrm{m}^{-2} \cdot \mathrm{a}^{-1}\right)<\mathrm{MB}(4.31$ www.plant-ecology.com $\left.\mathrm{kg} \cdot \mathrm{m}^{-2} \cdot \mathrm{a}^{-1}\right)<\mathrm{CK}\left(4.42 \mathrm{~kg} \cdot \mathrm{m}^{-2} \cdot \mathrm{a}^{-1}\right)<\mathrm{HB}(4.56$ $\left.\mathrm{kg} \cdot \mathrm{m}^{-2} \cdot \mathrm{a}^{-1}\right)$, 且与对照相比, LB和MB处理分别降低 $8.34 \% 、 1.48 \%, \mathrm{HB}$ 处理则增加 $4.71 \%$; 林分 4 中 LB $\left(4.34 \mathrm{~kg} \cdot \mathrm{m}^{-2} \cdot \mathrm{a}^{-1}\right)<$ MB $\left(4.46 \mathrm{~kg} \cdot \mathrm{m}^{-2} \cdot \mathrm{a}^{-1}\right)<$ HB $(4.74$ $\left.\mathrm{kg} \cdot \mathrm{m}^{-2} \cdot \mathrm{a}^{-1}\right)<\mathrm{CK}\left(4.75 \mathrm{~kg} \cdot \mathrm{m}^{-2} \cdot \mathrm{a}^{-1}\right)$, 与对照相比, LB、MB处理的土壤呼吸年总量分别降低 $7.98 \%$ 、 $5.77 \%$, HB则增加 $0.86 \%$ 。

\section{3 讨论}

\section{1 生物质炭添加对毛竹林土壤呼吸激发效应和 持效性的影响}

土壤激发效应是指各种有机质添加等处理引起 土壤有机质周转强烈的短期改变(Kuzyakov et al., 2000), 土壤中添加生物质炭后激发原位土壤碳排 放增加(正激发效应)或激发原位土壤碳排放降低(负 激发效应)。生物质炭添加对土壤的激发效应因土壤 类型和养分的不同对土壤呼吸的影响存在着诸多争 议, 主要包括促进(Luo et al., 2011)、抑制(Jones et al., 2011; Zimmerman et al., 2011)和无影响(Liu et al., 2016)。生物质炭添加后土壤碳矿化较对照明显增加, 

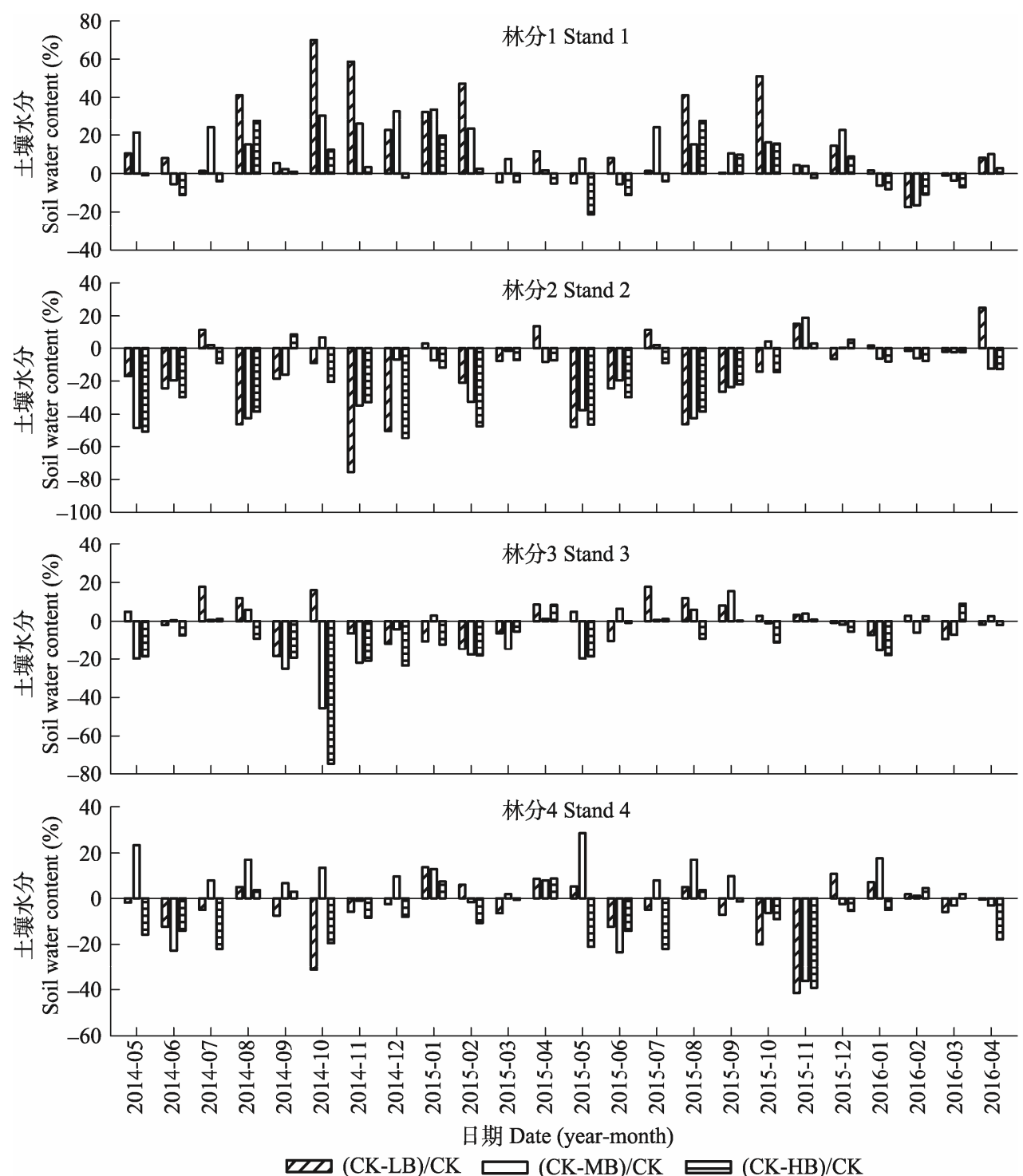

图3 生物质炭添加对毛竹林土壤水分含量月动态的影响。 $(\mathrm{CK}-\mathrm{LB}) / \mathrm{CK} 、(\mathrm{CK}-\mathrm{MB}) / \mathrm{CK} 、(\mathrm{CK}-\mathrm{HB}) / \mathrm{CK}$ 分别表示低、中、高 生物质炭添加处理下土壤水分含量较对照降低(负的表示增加)的百分比。缩写同图2。

Fig. 3 The effects of biochar addition on monthly soil moisture in Phyllostachys edulis forest stands. The $Y$-axis means the percent of soil moisture difference by biochar addition to the control. (CK-LB)/CK, $(\mathrm{CK}-\mathrm{MB}) / \mathrm{CK},(\mathrm{CK}-\mathrm{HB}) / \mathrm{CK}$ mean the decreased (minus value indicates a increase) percent of soil moisture on LB, MB and HB treatment, compared with control treatment. The abbreviations are the same as in Fig. 2.

这是因为生物质炭快速利用生物质炭自身的易溶碳 组分，没有激发原位土壤有机质的碳排放(Cross \& Sohi, 2011); 降低原位土壤碳矿化可能是因为增强 了原位土壤易溶碳库的稳定性。本研究发现生物质 炭添加明显降低毛竹林土壤呼吸速率, 与对照相比, LB 处理降低 $2.33 \%-54.72 \%$, MB 处理降低 $1.28 \%-$ $44.2 \%, \mathrm{HB}$ 处理则是降低了 $0.09 \%-39.22 \%$ (图1), 均 呈现负激发效应，这与Novak等(2010)和Spokas等 (2010)的研究结果一致。生物质炭添加降低毛竹林 土壤呼吸的原因可能是生物质炭表面孔隙结构吸收 了原位土壤有机质、促进原位土壤团聚体的稳定性,
同时土壤微生物因适应环境而发生变化(Herath et al., 2015); 低生物质炭添加因环境改变降低土壤碳 矿化明显，高生物质炭添加处理下微生物快速利用 生物质炭自身易溶养分而促进碳矿化，同时弥补因 适应环境而降低的碳矿化, 致使添加剂量与土壤呼 吸降低程度呈非线性关系(图7), 也可能因为非生物 因素(如降水)或生物质炭添加增加水分而使土壤中 碳酸盐含量增加(Bruun et al., 2014)。总之, 一方面 适宜的温度和水分能促进微生物生长和碳排放, 另 一方面土壤中添加生物质炭的激发方向和程度随着 土壤和生物炭类型而发生变化(Major et al., 2010)。 


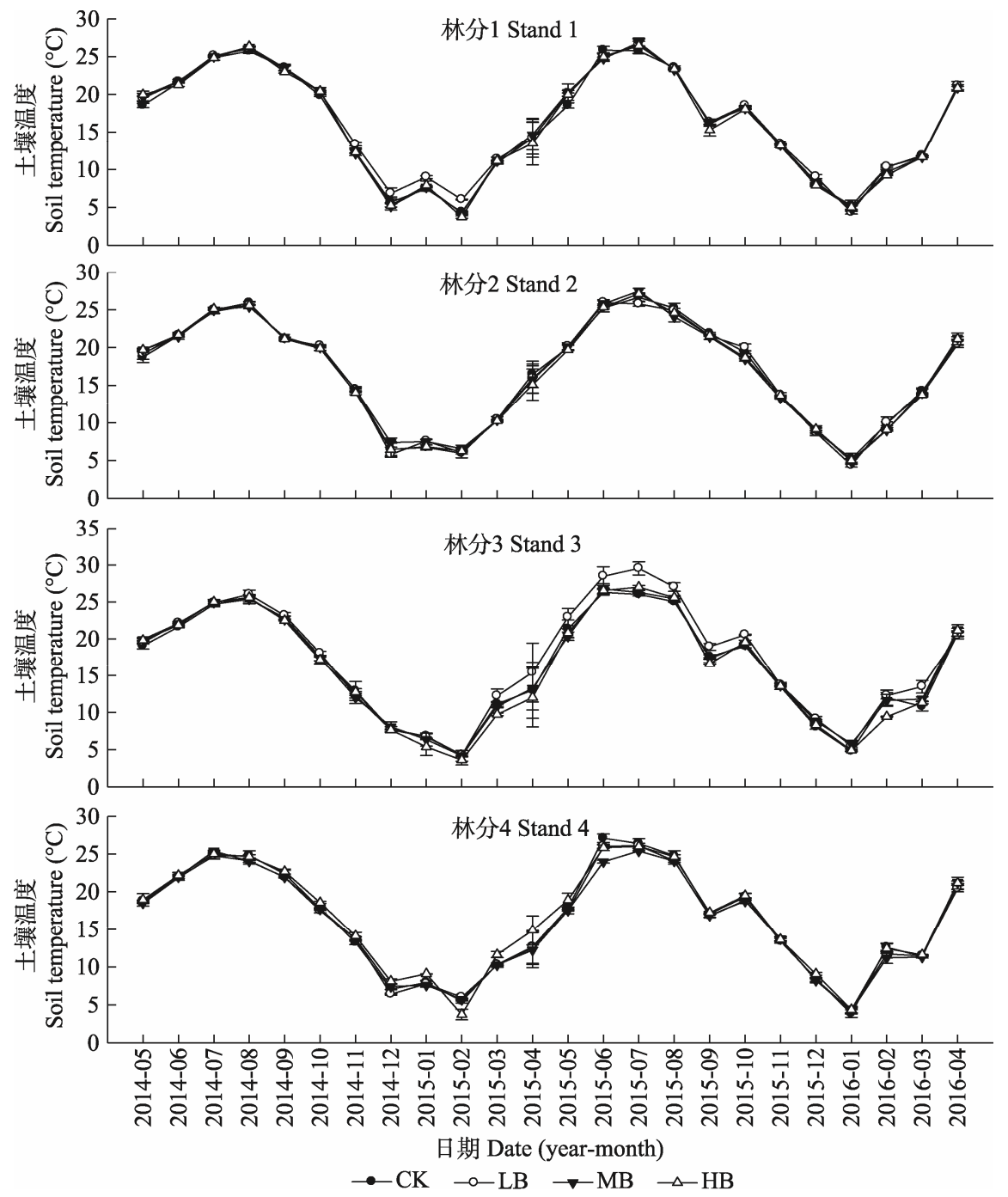

图4 生物质炭添加下毛竹林土壤温度月动态特征。 $\mathrm{CK}$, 对照; $\mathrm{LB}$, 低生物质炭施加 $\left(5 \mathrm{t} \cdot \mathrm{hm}^{-2}\right) ; \mathrm{HB}$, 高生物质炭施加 $\left(20 \mathrm{t} \cdot \mathrm{hm}^{-2}\right) ; \mathrm{MB}$ ，中生物质炭施加 $\left(10 \mathrm{t} \cdot \mathrm{hm}^{-2}\right)$ 。

Fig. 4 The characteristics of monthly soil temperaturein Phyllostachys edulis forest stands with different biochar addition treatments. CK, control; LB, low rate of biochar addition $\left(5 \mathrm{t} \cdot \mathrm{hm}^{-2}\right)$; HB, high rate of biochar addition $\left(20 \mathrm{t} \cdot \mathrm{hm}^{-2}\right)$; MB, medium rate of biochar addition $\left(10 \mathrm{t} \cdot \mathrm{hm}^{-2}\right)$.

表3 生物质炭添加下毛竹林土壤呼吸速率 $(y)$ 与土壤温度 $(x)$ 、水分 $(W)$ 关系的拟合方程

Table 3 Regression models of soil respiration $(y)$ with soil temperature $(x)$ and moisture $(W)$ in Phyllostachys edulis forest stands with different biochar addition treatments

\begin{tabular}{|c|c|c|c|c|c|c|}
\hline \multirow{2}{*}{$\begin{array}{l}\text { 林分 } \\
\text { Stand }\end{array}$} & \multicolumn{3}{|l|}{ CK } & \multicolumn{2}{|l|}{ LB } & \multirow[b]{2}{*}{$p$} \\
\hline & 拟合方程 Fitted equation & $R^{2}$ & $p$ & 拟合方程 Fitted equation & $R^{2}$ & \\
\hline 1 & $y=0.025 \mathrm{e}^{0.095 x}\left(2.609 W-0.066 W^{2}\right)$ & 0.80 & *** & $y=0.067 \mathrm{e}^{0.097 x}\left(0.523 W-0.012 W^{2}\right)$ & 0.79 & ** \\
\hline 2 & $y=0.029 \mathrm{e}^{0.088 x}\left(2.372 W-0.056 W^{2}\right)$ & 0.76 & ** & $y=0.112 \mathrm{e}^{0.093 x}\left(0.437 W-0.010 W^{2}\right)$ & 0.69 & ** \\
\hline 3 & $y=0.018 \mathrm{e}^{0.085 x}\left(3.968 W-0.096 W^{2}\right)$ & 0.72 & ** & $y=0.098 \mathrm{e}^{0.072 x}\left(0.819 W-0.020 W^{2}\right)$ & 0.72 & ** \\
\hline 4 & $y=0.030 \mathrm{e}^{0.077 x}\left(2.828 W-0.060 W^{2}\right)$ & 0.73 & ** & $y=0.021 \mathrm{e}^{0.093 x}\left(0.387 W-0.068 W^{2}\right)$ & 0.80 & ** \\
\hline \multirow{2}{*}{$\begin{array}{l}\text { 林分 } \\
\text { Stand }\end{array}$} & MB & \multicolumn{4}{|c|}{$\mathrm{HB}$} & \\
\hline & 拟合方程 Fitted equation & $R^{2}$ & $p$ & 拟合方程 Fitted equation & $R^{2}$ & $p$ \\
\hline 1 & $y=0.080 \mathrm{e}^{0.086 x}\left(0.653 W-0.012 W^{2}\right)$ & 0.65 & ** & $y=0.091 \mathrm{e}^{0.083 x}\left(0.510 W-0.008 W^{2}\right)$ & 0.65 & ** \\
\hline 2 & $y=0.024 \mathrm{e}^{0.091 x}\left(1.776 W-0.035 W^{2}\right)$ & 0.76 & ** & $y=0.096 \mathrm{e}^{0.078 x}\left(0.769 W-0.017 W^{2}\right)$ & 0.71 & ** \\
\hline 3 & $y=0.009 \mathrm{e}^{0.086 x}\left(6.901 W-0.157 W^{2}\right)$ & 0.73 & ** & $y=0.032 \mathrm{e}^{0.079 x}\left(2.237 W-0.049 W^{2}\right)$ & 0.74 & ** \\
\hline 4 & $y=0.062 \mathrm{e}^{0.090 x}\left(1.059 W-0.025 W^{2}\right)$ & 0.69 & ** & $y=0.015 \mathrm{e}^{0.095 x}\left(3.825 W-0.087 W^{2}\right)$ & 0.86 & ** \\
\hline
\end{tabular}

缩写同图4。

The abbreviations are the same as in Fig. 4.

www.plant-ecology.com 

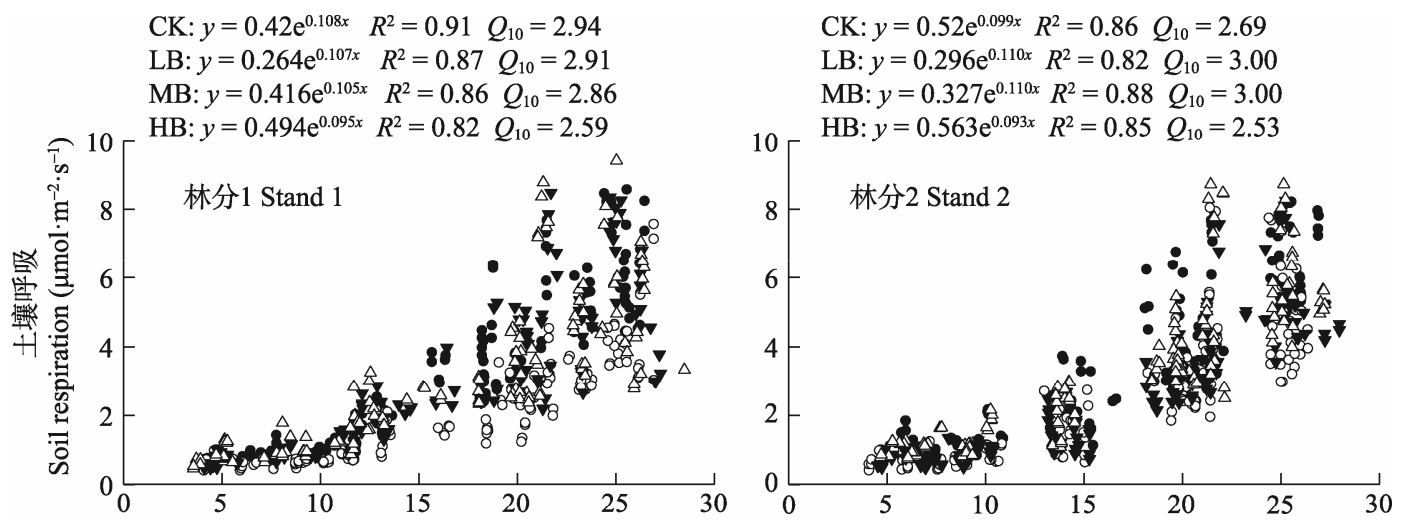

CK: $y=0.474 \mathrm{e}^{0.100 x} R^{2}=0.82 \quad Q_{10}=2.69$

LB: $y=0.418 \mathrm{e}^{0.096 x} R^{2}=0.83 \quad Q_{10}=2.61$

MB: $y=0.398 \mathrm{e}^{0.106 x} R^{2}=0.85 \quad Q_{10}=2.89$

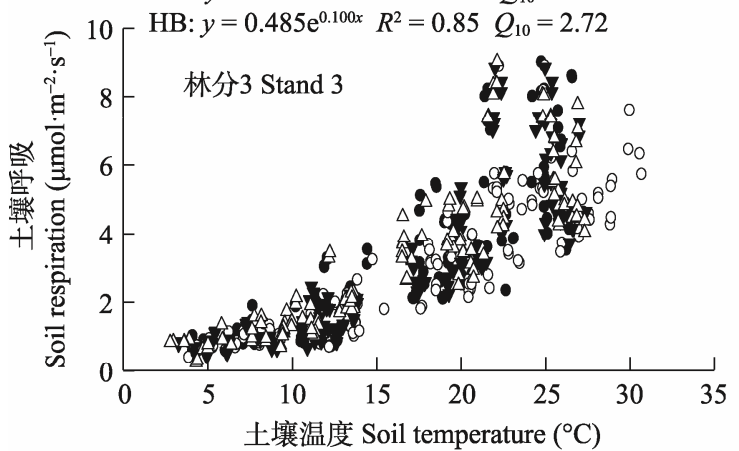

CK: $y=0.615 \mathrm{e}^{0.091 x} R^{2}=0.81 \quad Q_{10}=2.48$

LB: $y=0.446 \mathrm{e}^{0.104 x} R^{2}=0.85 \quad Q_{10}=2.83$

MB: $y=0.449 \mathrm{e}^{0.105 x} R^{2}=0.80 Q_{10}=2.86$

10 HB: $y=0.412 \mathrm{e}^{0.111 x} R^{2}=0.90 Q_{10}=3.03$

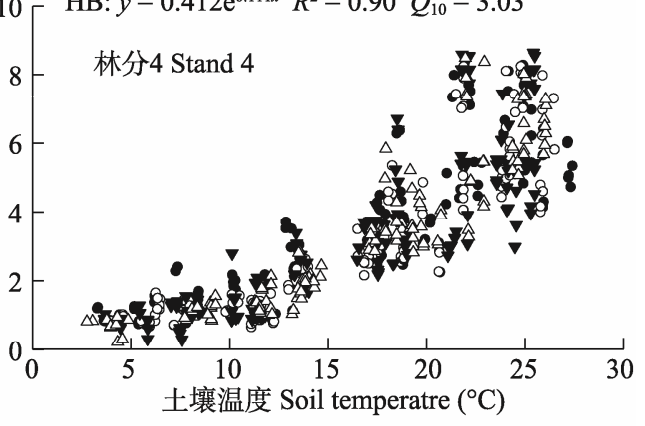

- $\mathrm{CK} \circ \mathrm{LB} \quad \mathrm{MB} \triangle \mathrm{HB}$

图5 生物质炭添加下毛竹林土壤呼吸速率和土壤温度的关系。CK, 对照; $\mathrm{LB}$, 低生物质炭施加 $\left(5 \mathrm{t} \cdot \mathrm{hm}^{-2}\right)$; $\mathrm{HB}$, 高生物质炭施 加 $\left(20 \mathrm{t} \cdot \mathrm{hm}^{-2}\right) ; \mathrm{MB}$ ，中生物质炭施加 $\left(10 \mathrm{t} \cdot \mathrm{hm}^{-2}\right)$ 。 $Q_{10}$ 土壤呼吸温度敏感性系数。

Fig. 5 Relationships between soil respiration and soil temperature in Phyllostachys edulis forest stands with different biochar addition treatments. CK, control; LB, low rate of biochar addition $\left(5 \mathrm{t} \cdot \mathrm{hm}^{-2}\right)$; HB, high rate of biochar addition $\left(20 \mathrm{t} \cdot \mathrm{hm}^{-2}\right)$; MB, medium rate of biochar addition $\left(10 \mathrm{t} \cdot \mathrm{hm}^{-2}\right) . Q_{10}$, soil temperature sensitivity.
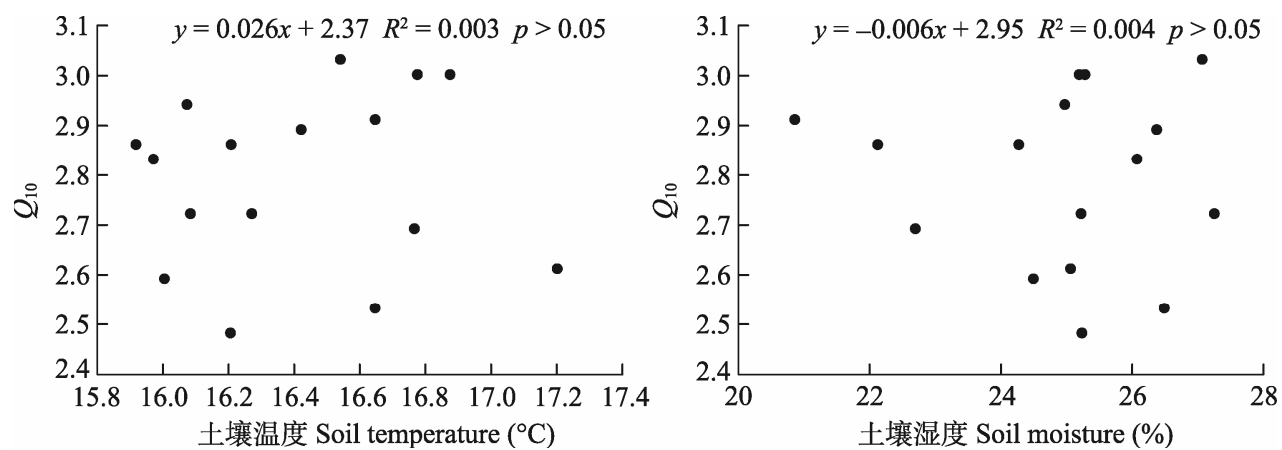

图6 生物质炭添加下毛竹林土壤呼吸温度敏感性系数 $\left(Q_{10}\right)$ 与土壤温度、土壤水分的关系。

Fig. 6 Relationships of soil temperature sensitivity $\left(Q_{10}\right)$ with soil temperature and moisture in Phyllostachys edulis forest stands with biochar addition.

生物质炭添加对土壤呼吸速率的季节变化影 响不同(图2; 表2)。本研究中生物质炭添加下土壤呼 吸速率均呈单峰模式, 土壤呼吸速率均在6-7月最高 (除林分1中LB处理是8月份最高外), 1月或2月最低; 生物质炭添加对生长季土壤呼吸速率的影响较非生 长季明显, 主要是因为生长季水分适宜(雨季), 土 壤温度相对适合微生物活性、根系生长和植物的发
育阶段(吴君君等, 2014); 生长季温度相对较高时, 根呼吸和微生物呼吸相对旺盛, 生物质炭添加增加 微生物活性、微生物丰度和根系生物量(Mitchell et $a l ., 2015)$; 在非生长季当温度较低时, 土壤呼吸速 率主要受生化反应限制, 生物质炭添加对根系呼吸 和微生物活性影响相对较小, 因此, 生物质炭添加 处理下非生长季碳排放对年碳排放的贡献相对较小。 

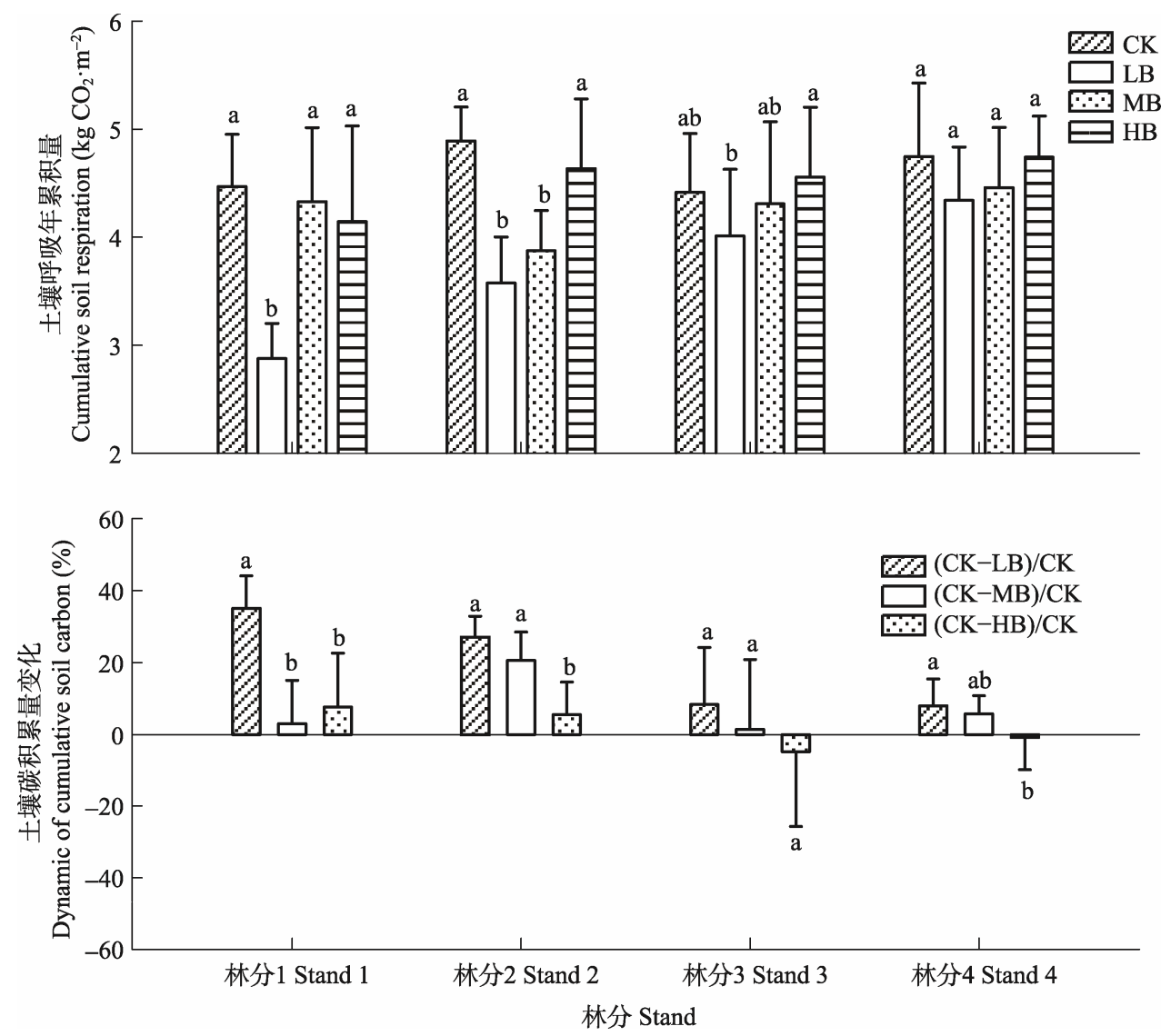

图7 生物质炭添加对毛竹林土壤碳年累积排放的影响 (平均值沶准偏差)。不同小写字母表示相同林分不同处理间差异显 著。 $\mathrm{CK}$, 对照; $\mathrm{LB}$ ，低生物质炭施加 $\left(5 \mathrm{t} \cdot \mathrm{hm}^{-2}\right) ; \mathrm{MB}$ ，中生物质炭施加 $\left(10 \mathrm{t} \cdot \mathrm{hm}^{-2}\right)$; HB，高生物质炭施加 $\left(20 \mathrm{t} \cdot \mathrm{hm} \mathrm{m}^{-2}\right)$; $(\mathrm{CK}-\mathrm{LB}) / \mathrm{CK} 、(\mathrm{CK}-\mathrm{MB}) / \mathrm{CK} 、(\mathrm{CK}-\mathrm{HB}) / \mathrm{CK}$ 分别表示低、中、高生物质炭添加处理中土壤碳年累积排放量较对照降低的比重。 Fig. 7 The effects of biochar addition on annual cumulative soil respiration in Phyllostachys edulis forest stands (mean $\pm S D)$. Different lowercase letters indicate significant differences among biochar treatments within forest stands. CK, control; LB, low rate of biochar addition $\left(5 \mathrm{t} \cdot \mathrm{hm}^{-2}\right)$; MB, medium rate of biochar addition $\left(10 \mathrm{t} \cdot \mathrm{hm}^{-2}\right)$; HB, high rate of biochar addition $\left(20 \mathrm{t} \cdot \mathrm{hm} \mathrm{m}^{-2}\right)$. $(\mathrm{CK}-\mathrm{LB}) / \mathrm{CK},(\mathrm{CK}-\mathrm{MB}) / \mathrm{CK},(\mathrm{CK}-\mathrm{HB}) / \mathrm{CK}$ mean the decrease percent of annual cumulative soil respiration on LB, MB and $\mathrm{HB}$ treatment, compared with control treatment.

生物质炭添加对毛竹林土壤呼吸速率的后续影 响与添加剂量相关。研究表明生物质炭添加到土壤 后，自身碳矿化随着时间逐渐降低(Ameloot et al., $2013)$; 生物质炭添加的起始阶段, $2 \%-20 \%$ 的生物质 炭自身碳在2-60天矿化, 随后矿化率降低。如Major 等(2010)研究表明生物质炭添加对土壤呼吸第一年 的影响较第二年大, 生物质炭自身易溶碳矿化 $75 \%$ 集中在第一年且随着时间的延长逐渐降低。本研究 中低生物质炭添加处理降低土壤呼吸的程度随着时 间的延长略微降低, 中、高生物质炭添加处理降低土 壤呼吸程度随时间的延长略微增加, 这可能是因为 土壤微生物与生物质炭自身易溶养分相互作用或适 应环境程度与生物质炭添加剂量有关。因此, 生物 质炭与土壤互作对土壤碳矿化持久性的影响因素 需要进一步深入研究。

\section{2 生物质炭添加对土壤温度、水分含量及温度敏 感性的影响}

土壤温度、土壤水分是影响土壤 $\mathrm{CO}_{2}$ 通量的主 导因子，它们通过对调落物分解过程、微生物活性、 根系生长及光合作用调节而影响土壤呼吸速率 (Lellei-Kovács et al., 2016)。本研究中, 生物质炭添 加增加土壤水分含量(图3), 可能是因为生物质炭添 加通过增加田间持水量孔隙 $(30 \mu \mathrm{m})$ 和永久萎蒸点 间孔隙 $(0.2 \mu \mathrm{m})$ 的比重而增加土壤有效水分(Hardie et al., 2014)。对照和生物质炭处理下土壤呼吸与温 度呈显著的指数函数关系(图5), 因为土壤温度通过 影响微生物呼吸、根系生长和养分矿化等影响土壤 呼吸; 土壤呼吸与水分含量均无显著相关关系, 可 能是因为毛竹林土壤含水量相对适宜, 土壤含水量 不是土壤呼吸的限制因子, 高浓度生物质炭添加虽 
增加土壤水分含量但对土壤呼吸的解释量较小 $(\mathrm{He}$ et al., 2016)。土壤呼吸速率与温度、水分加和呈显 著相关关系(表3), 说明土壤水分通过土壤温度间接 影响土壤呼吸速率; 生物质炭添加明显增加了土壤 水分含量, 尤其是增加土壤有效水分容量的 $0.3 \%-1.3 \%$ 时, 生物质炭添加增加的土壤水分含量 引起传导性和电解质损失而形成一个相对高温的环 境，间接影响土壤呼吸速率(Ulyett et al., 2014)。

温度和水分等环境因子通过土壤生物和底物间 接影响土壤呼吸的温度敏感性(杨庆朋等, 2011), 通 常土壤含水量增加会提高土壤呼吸的温度敏感性, 在一定范围内, 随着土壤含水量的升高, 土壤呼吸 对温度的响应会更为剧烈(Almagro et al., 2009)。本 研究中生物质炭添加增加土壤水分含量和改变土壤 呼吸底物等引起土壤呼吸的温度敏感性变化(图5), 这可能是因为: 1)环境因子变化, 生物质炭添加后 土壤水分含量增加直接影响温度敏感性;2)土壤呼 吸底物变化, 生物质炭自身养分含量或生物质炭表 面物质抑制原位土壤有机碳和根系分解, 或生物质 炭添加引起温度变化(He et al., 2016); 3)土壤根系 (生物)变化, 生物质炭添加使土壤根系增加, 而根 呼吸是土壤呼吸的主要组成部分且具有较高的温度 敏感性(Saiz et al., 2006)。He等(2016)研究表明生物 质炭自身孔隙吸收 $\mathrm{CO}_{2}$, 生物质炭添加降低土壤温 度变动, 在一定程度上解释土壤温度敏感性变化。

Suseela等(2012)研究表明春、秋季 $Q_{10}$ 与平均年土壤 水分和平均年土壤温度呈正相关关系; 夏季 $Q_{10}$ 与 土壤温度和水分不相关。本研究中, $Q_{10}$ 与土壤平均 年温度和水分不相关(图6), 可能是因为土壤水分含 量变化没有超出毛竹林温度敏感性响应的阈值(刘 殿军等, 2016); 当土壤水分含量低于 $0.11 \mathrm{~m}^{3} \cdot \mathrm{m}^{-3}$ 或 大于田间持水量时, $Q_{10}$ 降低, 土壤水分胁迫影响土 壤温度敏感性(Jassal et al., 2008)。总之, 土壤含水量 相对适宜的时候温度敏感性最高, 而土壤温度敏感 性在含水量较低或较高时都会发生变化, 变化方向 和幅度与土壤类型紧密相关(杨庆朋等, 2011)。

\section{3 生物质炭添加对土壤碳排放的影响}

生物质炭对土壤 $\mathrm{CO}_{2}$ 排放(土壤呼吸) 的影响因 素包括微生物的生物降解和环境的非有机碳部分的 非生物因素(Jones et al., 2011); 生物质炭添加能改 变土壤物理化学性质和微生物繁殖的环境, 并影响 土壤微生物活性和群落结构(Liu et al., 2016)。本
研究中生物质炭添加剂量对土壤碳排放的影响显著 (图 7), 与Chen等(2017)和Bamminger等(2014)的研 究吻合, 即生物质炭添加后累积 $\mathrm{CO}_{2}$ 排放与对照相 比降低了 $2 \%-56 \%$, 且随着添加量的增加而下降, 因为生物质炭添加对土壤碳排放的生物因子影响明 显, 尤其生物质炭添加明显增加根际区域面积, 生 物质炭自身养分或增加的水分吸引植物根系; 同时 可能因其降低根系新陈代谢活动而降低根系呼吸 (Prendergast-Miller et al., 2014)。因此, 大规模野外 试验之前有必要粗略地评估生物质炭添加效果, 尤 其是土壤养分状况、生物质炭类型和森林经营措施 (Kammann et al., 2011)。

生物质炭添加对土壤碳排放的影响与非生物因 子关系密切, 生物质炭添加的负激发效应与土壤碳 酸盐含量紧密相关(Verheijen et al., 2014)。用 ${ }^{14} \mathrm{C}$ 标 记的生物质炭研究表明在培养的土壤中碳酸盐含量 与底物总 $\mathrm{CO}_{2}$ 排放紧密相关(Bruun et al., 2014)。试 验条件也是生物质炭添加影响土壤呼吸的一个重要 因素: 在野外, 生物质炭添加增加土壤团聚体的稳 定性(Hardie et al., 2014), 有机物质被大团聚体保护, 或被土壤矿化颗粒束缚, 不易被土壤微生物直接利 用(Liang et al., 2010)。本研究中野外原位土壤添加 生物质炭后土壤通过有机质吸收过程和物理保护提 高土壤碳库吸存, 降低土壤碳排放(图7)。有研究表 明土壤早期培养阶段的碳释放明显来自碳酸盐; 尽 管碳酸盐能解释 $\mathrm{CO}_{2}$ 的非生物释放, 其他的非生物 过程如生物质炭表面的非生物氧化也很重要(Bruun et al., 2014)。

土壤呼吸除受到非生物因子影响外, 还受到土 壤微生物、植被类型等生物因子的影响。生物质炭 添加改变微生物群落, 因为其通过对底物有效养分 和 $\mathrm{pH}$ 值的改变而改变微生物群落组成(Fierer et al., 2009)。本研究中, 生物质炭添加明显改变了微生物 的群落结构, 中、高生物质炭处理增加了细菌和总 微生物群落生物量(未发表)。生物质炭与根系相互 作用, 增加根系生物量和根长密度, 植物根系对生 物质炭添加因水分含量不同而响应不同(Verheijen et al., 2014)。

基金项目国家自然科学基金 (31600492和 31670607)和中央级公益性科研院所基本科研业务 费专项资金(CAFYBB2016SY006、CAFBB2014Q- 


\section{A008和CAFRISF2013002)。}

\section{参考文献}

Almagro M, López J, Querejeta JI, Martínez-Mena M (2009). Temperature dependence of soil $\mathrm{CO}_{2}$ efflux is strongly modulated by seasonal patterns of moisture availability in a Mediterranean ecosystem. Soil Biology \& Biochemistry, 41, 594-605.

Ameloot N, Graber ER, Verheijen FGA, de Neve S (2013). Interactions between biochar stability and soil organisms: Review and research needs. European Journal of Soil Science, 64, 379-390.

Bamminger C, Marschner B, Jüschke E (2014). An incubation study on the stability and biological effects of pyrogenic and hydrothermal biochar in two soils. European Journal of Soil Science, 65, 72-82.

Bruun S, Clauson-Kaas S, Bobuĭská L, Thomsen IK (2014). Carbon dioxide emissions from biochar in soils: Role of clay, microorganisms and carbonates. European Journal of Soil Science, 65, 52-59.

Chen JH, Li SH, Liang CF, Xu QF, Li YC, Qin H, Fuhrmann JF (2017). Response of microbial community structure and function to short-term biochar amendment in an intensively managed bamboo (Phyllostachys praecox) plantation soil: Effect of particle size and addition rate. Science of the Total Environment, 574, 24-33.

Cross A, Sohi SP (2011). The priming potential of biochar products in relation to labile carbon contents and soil organic matter status. Soil Biology \& Biochemistry, 43, 2127-2134.

DeLuca TH, MacKenzie MD, Gundale MJ, Holben WE (2006). Wildfire-produced charcoal directly influences nitrogen cycling in Ponderosa pine forests. Soil Science Society of America Journal, 70, 448-453.

Fierer N, Strickland MS, Liptzin D, Bradford MA, Cleveland CC (2009). Global patterns in belowground communities. Ecology Letters, 12, 1238-1249.

Forbes MS, Raison RJ, Skjemstad JO (2006). Formation, transformation and transport of black carbon (charcoal) in terrestrial and aquatic ecosystems. Science of the Total Environment, 370, 190-206.

Ge XG, Zhou BZ, Xiao WF, Wang XM, Cao YH (2016). Priming effect of biochar addition on soil carbon emission: A review. Ecology and Environmental Sciences, 25, 339-345. (in Chinese with English abstract) [葛晓改, 周 本智, 肖文发, 王小明, 曹永慧 (2016). 生物质炭输入 对土壤碳排放的激发效应研究进展. 生态环境学报, 25 , 339-345.]

Hardie M, Clothier B, Bound S, Oliver G, Close D (2014). Does biochar influence soil physical properties and soil water availability? Plant and Soil, 376, 347-361.

Hashimoto S, Carvalhais N, Ito A, Migliavacca M, Nishina K, Reichstein M (2015). Global spatiotemporal distribution of soil respiration modeled using a global database. Biogeosciences, 12, 4121-4132.

He XH, Du ZL, Wang YD, Lu N, Zhang QZ (2016). Sensitivity of soil respiration to soil temperature decreased under deep biochar amended soils in temperate croplands. Applied Soil Ecology, 108, 204-210.

Herath HMSK, Camps-Arbestain M, Hedley MJ, Kirschbaum MUF, Wang T, van Hale R (2015). Experimental evidence for sequestering $\mathrm{C}$ with biochar by avoidance of $\mathrm{CO}_{2}$ emissions from original feedstock and protection of native soil organic matter. Global Change Biology Bioenergy, 7, 512-526.

Hughes RF, Kauffman JB, Jaramillo VJ (1999). Biomass, carbon, and nutrient dynamics of secondary forests in a humid tropical region of méxico. Ecology, 80, 1892-1907.

Jassal RS, Black TA, Novak MD, Gaumont-Guay D, Nesic Z (2008). Effect of soil water stress on soil respiration and its temperature sensitivity in an 18-year-old temperate Douglas-fir stand. Global Change Biology, 14, 1305-1318.

Jones DL, Murphy DV, Khalid M, Ahmad W, Edwards-Jones G, DeLuca TH (2011). Short-term biochar-induced increase in soil $\mathrm{CO}_{2}$ release is both biotically and abiotically mediated. Soil Biology \& Biochemistry, 43, 1723-1731.

Kammann CI, Linsel S, Gößling JW, Koyro HW (2011). Influence of biochar on drought tolerance of Chenopodium quinoa Willd and on soil-plant relations. Plant and Soil, $345,195-210$.

Kuzyakov Y, Friedel JK, Stahr K (2000). Review of mechanisms and quantification of priming effects. Soil Biology \& Biochemistry, 32, 1485-1498.

Lal R, Follett RF, Stewart BA, Kimble JM (2007). Soil carbon sequestration to mitigate climate change and advance food security. Soil Science, 172, 943-956.

Lehmann J, Gaunt J, Rondon M (2006). Bio-char sequestration terrestrial ecosystems-A review. Mitigation and Adaptation Strategies for Global Change, 11, 403-427.

Lellei-Kovács E, Botta-Dukát Z, de Dato G, Estiarte M, Guidolotti G, Kopittke GR, Kovács-Láng E, Kröel-Dulay G, Larsen KS, Peñuelas J, Smith AR, Sowerby A, Tietema A, Schmidt IK (2016). Temperature dependence of soil respiration modulated by thresholds in soil water availability across European shrubland ecosystems. Ecosystems, 19, $1460-1477$.

Liang BQ, Lehmann J, Sohi SP, Thies JE, O’Neill B, Trujillo L, Gaunt J, Solomon D, Grossman J, Neves EG, Luizão FJ (2010). Black carbon affects the cycling of non-black carbon in soil. Organic Geochemistry, 41, 206-213.

Liu DJ, Zhang JX, Lu Q, Li X, Wu Z (2016). Effects of rain supplementation on the temperature sensitivity of soil respiration in Nitraria sphaerocarpa community in a hyperarid area of Dunhuang, China. Chinese Journal of Ecology, 35, 584-590. (in Chinese with English abstract)

www.plant-ecology.com 
[刘殿君, 张金金金, 卢琦, 李旭, 武哲 (2016). 极端干旱 区增雨对泡泡刺(Nitraria sphaerocarpa)群落土壤呼吸 温度敏感性的影响. 生态学杂志, 35, 584-590.]

Liu XY, Zheng JF, Zhang DX, Cheng K, Zhou HM, Zhang AF, Li LQ, Joseph S, Smith P, Crowley D, Kuzyakov Y, Pan GX (2016). Biochar has no effect on soil respiration across Chinese agricultural soils. Science of the Total Environment, 554-555, 259-265.

Luo Y, Durenkamp M, de Nobili M, Lin Q, Brookes PC (2011). Short term soil priming effects and the mineralization of biochar following its incorporation to soils of different $\mathrm{pH}$. Soil Biology \& Biochemistry, 43, 2304-2314.

Major J, Rondon M, Molina D, Riha SJ, Lehmann J (2010). Maize yield and nutrition during 4 years after biochar application to a Colombian savanna oxisol. Plant and Soil, $333,117-128$.

Marland E, Marland G (2003). The treatment of long-lived, carbon-containing products in inventories of carbon dioxide emissions to the atmosphere. Environmental Science \& Policy, 6, 139-152.

Mitchell PJ, Simpson AJ, Soong R, Simpson MJ (2015). Shifts in microbial community and water-extractable organic matter composition with biochar amendment in a temperate forest soil. Soil Biology \& Biochemistry, 81, 244254.

Novak JM, Busscher WJ, Watts DW, Laird DA, Ahmedna MA, Naiandou MAS (2010). Short-term $\mathrm{CO}_{2}$ mineralization after additions of biochar and switchgrass to a typic kandiudult. Geoderma, 154, 281-288.

Prendergast-Miller MT, Duvall M, Sohi SP (2014). Biocharroot interactions are mediated by biochar nutrient content and impacts on soil nutrient availability. European Journal of Soil Science, 65, 173-185.

Saiz G, Byrne KA, Butterbach-Bahl K, Kiese R, Blujdea V, Farrell EP (2006). Stand age-related effects on soil respiration in a first rotation Sitka spruce chronosequence in central Ireland. Global Change Biology, 12, 1007-1020.

Sohi SP, Krull E, Lopez-Capel E, Bol R (2010). A review of biochar and its use and function in soil. In: Donald LS ed. Advances in Agronomy. Academic Press, Burlington, USA. 47-82.

Spokas KA, Baker JM, Reicosky DC (2010). Ethylene: Potential key for biochar amendment impacts. Plant and Soil, 333, 443-452.

Suseela V, Conant RT, Wallenstein MD, Dukes JS (2012). Effects of soil moisture on the temperature sensitivity of heterotrophic respiration vary seasonally in an old-field climate change experiment. Global Change Biology, 18, 336-348.

Tammeorg P, Simojoki A, Mäkelä P, Stoddard FL, Alakukku L, Helenius J (2014). Short-term effects of biochar on soil properties and wheat yield formation with meat bone meal and inorganic fertiliser on a boreal loamy sand. Agriculture, Ecosystems and Environment, 191, 108-116.

Thomas SC, Gale N (2015). Biochar and forest restoration: A review and meta-analysis of tree growth responses. New Forest, 46, 931-946.

Ulyett J, Sakrabani R, Kibblewhite M, Hann M (2014). Impact of biochar addition on water retention, nitrification and carbon dioxide evolution from two sandy loam soils. European Journal of Soil Science, 65, 96-104.

Verheijen FGA, Graber ER, Ameloot N, Bastos AC, Sohi S, Knicker H (2014). Biochars in soils: New insights and emerging research needs. European Journal of Soil Science, 65, 22-27.

Wardle DA, Nilsson MC, Zackrisson O (2008). Fire-derived charcoal causes loss of forest humus. Science, 320, 629.

Wu JJ, Yang ZJ, Liu XF, Xiong DC, Lin WS, Chen CQ, Wang $\mathrm{XH}$ (2014). Analysis of soil respiration and components in Castanopsis carlesii and Cunninghamia lanceolata plantations. Chinese Journal of Plant Ecology, 38, 45-53. (in Chinese with English abstract) [吴君君，杨智杰，刘小飞， 熊德成, 林伟盛, 陈朝琪, 王小红 (2014). 米槠和杉木 人工林土壤呼吸及其组分分析. 植物生态学报, $38,45-$ 53.]

$\mathrm{Xu}$ Y (2012). Research on Community Structure and Carbon Accumulation of Moso Bamboo Forest. PhD dissertation, Zhejiang University, Hangzhou. (in Chinese with English abstract) [徐涌 (2012). 毛竹林群落结构与碳积累规律 研究. 博士学位论文, 浙江大学, 杭州.]

Yang QP, Xu M, Liu HS, Wang JS, Liu LX, Chi YG, Zheng YP (2011). Impact factors and uncertainties of the temperature sensitivity of soil respiration. Acta Ecologica Sinica, 31, 2301-2311. (in Chinese with English abstract) [杨庆朋, 徐明, 刘洪升, 王劲松, 刘丽香, 迟永刚, 郑 云普 (2011). 土壤呼吸温度敏感性的影响因素和不确 定性. 生态学报, 31, 2301-2311.]

Yuste JC, Baldocchi DD, Gershenson A, Goldstein A, Misson L, Wong S (2007). Microbial soil respiration and its dependency on carbon inputs, soil temperature and moisture. Global Change Biology, 13, 2018-2035.

Zhou BZ (2006). Dynamics of Bamboo's Below-ground System Based on Minirhizotron Technique. PhD dissertation, Chinese Academy of Forestry, Beijing. (in Chinese with English abstract) [周本智 (2006). 基于小观察窗技术的竹林 地下系统动态研究. 博士学位论文, 中国林业科学研究 院, 北京.]

Zimmerman AR, Gao B, Ahn MY (2011). Positive and negative carbon mineralization priming effects among a variety of biochar-amended soils. Soil Biology \& Biochemistry, $43,1169-1179$. 


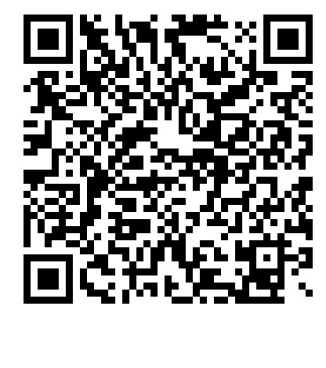

